fewer with combined chemo-radiation. Surgery was generally proceeded particularly in the patients who had responses or at least stable diseases to neoadjuvant treatment. Peri-operative outcomes after neoadjuvant treatment were superior compared to those of primary surgery whereas survival data were still inconsistent. Features which had or tended to have favorable prognosis were younger age, early-stage disease, response to neoadjuvant treatment, low pre-operative CA125, and optimal surgery. Among different modalities of neoadjuvant treatment which has become a frequent mode of treatment, neoadjuvant chemotherapy was more common than radiation therapy alone or chemoradiation.

Conclusion: Radiation therapy alone (of different modes) was used as a sole definitive modality of therapy particularly for early-stage diseases which limited to uterine body, cervix, with or without parametrial invasion. Among different modalities of neoadjuvant treatment, neoadjuvant chemotherapy was more common than radiation therapy alone or chemoradiation.

Poster (E25)

Endometrial Hyperplasia, Endometrial Intra-epithelial Neoplasia, and Endometrial Cancer

https://doi.org/10.3802/jgo.2021.32.S1.E25

\section{Mismatch repair deficiency and real-world outcomes of immunotherapy in patients with endometrial cancer}

\section{Sohyun Nam, Shin-Wha Lee, Dae-Yeon Kim, Dae-Shik Suh, Jong-Hyeok Kim, Yong-Man Kim, Young-Tak Kim, Joo-Hyun Nam, Jeong-Yeol Park ${ }^{*}$}

Asan Medical Center, Seoul, Korea (catgut1-0@hanmail.net)

Objective: To investigate the clinicopathologic characteristics and the oncologic outcomes of immunotherapy in patients with endometrial cancer according to mismatch repair deficient (MMRd)/microsatellite instability (MSI) status.

Methods: We conducted a single-center retrospective analysis and a total of 215 endometrial cancer patients who underwent MMRd/MSI tests between January 2011 and March 2021 were included.

Results: Of the total 215 patients, 169 patients (78.6\%) had mismatch repair proficient (MMRp)/microsatellite stable (MSS) tumors and 46 patients (21.4\%) had MMRd/MSI tumors. Between 2 groups, distribution of histologic types and pathological stage were not statistically different ( $\mathrm{p}=0.469$ and 0.431 , respectively). A total of 68 out of 215 patients were tested for programmed death-ligand 1 (PD-L1), and PD-L1 expression was observed in $51.9 \%$ in MMRp/MSS group and 85.7\% in MMRd/MSI group $(p=0.022)$. The progression-free survival and overall survival rates did not statistically differ between 2 groups. Eight patients were treated with pembrolizumab and objective response rate was $37.5 \%$, comprised of 3 partial responses. Sixteen patients received lenvatinib/pembrolizumab and objective response rate was 31.3, comprised of 3 complete responses and 2 partial responses. Grade 3 or higher adverse events occurred in $18.8 \%$ (3/16) of those treated with lenvatinib/pembrolizumab.

Conclusion: In patients with endometrial cancer, although MMRd/MSI is associated with a higher PD-L1 expression rate than MMRp/MSS, there was no effect on progression and overall survival. In clinical practice, immunotherapy was safe and efficacious in recurrent endometrial cancer.

Poster (E26)

Endometrial Hyperplasia, Endometrial Intra-epithelial Neoplasia, and Endometrial Cancer

https://doi.org/10.3802/jgo.2021.32.S1.E26

\section{Nomogram for microscopic lymph node metastasis in surgically staged endometrioid endometrial cancer patients}

Tae-Wook Kong,, Jayoun Kim, ${ }^{2}$ Joo-Hyuk Son, ${ }^{1}$ Jiheum Paek, Suk-Joon Chang, ${ }^{1, *}$ Hee-Sug Ryu ${ }^{1}$

'Division of Gynecologic Oncology, Department of Obstetrics and Gynecology, Ajou University School of Medicine, Suwon, Korea (drchang@ajou.ac.kr)

${ }^{2}$ Medical Research Collaborating Center, Seoul National University College of Medicine, Seoul, Korea

Objective: To establish a nomogram for predicting microscopic lymph node (LN) metastasis in surgically staged endometrioid endometrial cancer (EC) patients.

Methods: Clinicopathologic data of presumed earlystage endometrioid EC patients $(n=338)$ treated with lymphadenectomy between March 2000 and February 2018 were analyzed. The nomogram was developed based on multivariate logistic regression analysis of preoperative clinicopathologic data. The predicted and discriminative ability of the nomogram were evaluated by a concordance index and calibration curve. The high-risk group was predefined as having a predicted probability of LN metastasis $>50 \%$.

Results: Thirty-four patients (10.1\%) had microscopic LN metastasis. Multivariate analysis identified cancer antigen-125, myometrial invasion on magnetic resonance imaging (MRI), cervical stromal invasion on MRI as independent prognostic factors associated with LN metastasis. The concordance index of the nomogram was 0.818 ( $95 \%$ confidence interval $=0.793-0.935)$, and calibration plots revealed good agreement between the observed probabilities and nomogram- 